\title{
Enquête
}

Archives de la revue Enquête

4 | 1996

La ville des sciences sociales

\section{Urbanités marseillaises}

Marseille, terrain des sciences sociales

Marseilles, a field for social sciences

\section{Pierre-Paul Zalio}

\section{(2) OpenEdition}

Journals

Édition électronique

URL : http://journals.openedition.org/enquete/903

DOI : 10.4000/enquete.903

ISSN : 1953-809X

Éditeur :

Cercom, Éditions Parenthèses

\section{Édition imprimée}

Date de publication : 1 novembre 1996

Pagination : 191-210

Référence électronique

Pierre-Paul Zalio, « Urbanités marseillaises », Enquête [En ligne], 4| 1996, mis en ligne le 12 juillet 2013, consulté le 19 avril 2019. URL : http://journals.openedition.org/enquete/903 ; DOI : 10.4000/ enquete.903

Ce document a été généré automatiquement le 19 avril 2019. 


\title{
Urbanités marseillaises
}

\author{
Marseille, terrain des sciences sociales \\ Marseilles, a field for social sciences
}

\author{
Pierre-Paul Zalio
}

1 L'histoire de la recherche urbaine à Marseille, de l'enquête de Jacques Loew, précurseur national, aux terrains contemporains, en passant par les travaux de Marcel Roncayolo, ceux des équipes de Paul-Henri Chombart de Lauwe, de Raymond Ledrut ou du CERFISE, semble témoigner en première lecture des évolutions du système administratif de recherche et de ruptures liées aux changements économiques, sociaux et politiques locaux, avec à chaque période des problématiques et taxinomies propres. De ce point de vue, les logiques successives de recherche paraissent devoir être réinscrites dans le cadre chronologique - pour partie singulier, pour partie reflet des scansions des politiques urbaines au niveau national - de l'histoire urbaine marseillaise ${ }^{1}$.

2 Néanmoins, le parcours d'un demi-siècle de recherche urbaine marseillaise que nous proposons ici, sans prétendre à l'exhaustivité, montre comment la diversité successive des problématiques de recherche, loin de se réduire à de simples ajustements à des conjonctures institutionnelles et idéologiques, constitue Marseille en laboratoire urbain, «Chicago français» tardif peut-être, mais qui en 1945 a trouvé ses sociologues². Ethnologie missionnaire, enquêtes chombartiennes ont ainsi constitué les premiers contrepoints des analyses fonctionnalistes des urbanistes. Finalement, la pluralité des orientations des recherches présentées ici atteste moins de l'éclatement ou de l'incertitude épistémologique d'une science urbaine prise au piège des singularités de terrain et de conjoncture, que d'une capacité (certes variable), face aux problématiques préconstituées par la demande sociale, à innover en terme de protocole d'observation (variation des échelles du quartier à l'aire métropolitaine, des situations d'interaction aux mobilités), de méthodes (du questionnaire de satisfaction au terrain ethnologique en passant par la géographie quantitative) et de catégorie d'analyse (le cas de la notion de centralité offrant un exemple remarquable). 
Depuis les années trente et l'élaboration du premier plan d'urbanisme par Jacques Greber 3, Marseille a été un terrain de recherches urbaines moins sociologiques que géographiques et urbanistiques ${ }^{4}$, stylisant de manière assez convergente les traits du contexte urbain autour de la particularité géographique du site, de la fonction portuaire et commerciale de la ville et des singularités de son plan. Entourée de collines, Marseille contient dans son vaste territoire sa banlieue, ses industries et sa campagne; isolée de l'arrière-pays, elle semble vouée à une fonction de port d'entrepôt et de transit. À la forme d'industrialisation spécifique du capitalisme négociant marseillais est attribué le contraste entre le développement du port et les retards de l'urbanisation, ainsi qu'un peuplement ouvrier et immigré nombreux et peu qualifiés. Enfin, la forme urbaine est caractérisée par la juxtaposition de quatre espaces qui communiquent mal entre eux : le port moderne au nord, la vieille ville infranchissable au centre, la ville « moderne » issue de l'agrandissement de 1666 au sud, et les vastes banlieues constituées, sous la poussée démographique ouvrière, par morcellement des propriétés rurales aux interstices des voies de communication. Les formes de cette urbanisation extensive et son peuplement, en d'autres termes la morphologie sociale, engendrent une situation où « le rapport entre une population aux revenus assez faibles et une surface énorme à entretenir, assainir et équiper, s'amenuise et rend pratiquement impossible la gestion municipale ${ }^{6} »$.

Par rapport à ces caractéristiques, les premières analyses fonctionnelles, contenues dans le plan présenté par Jacques Greber en 1933, prônent la construction de grands ensembles d'habitations bordant les pénétrantes et les rocades inscrites dans le plan, évitant ainsi la difficulté posée par le traitement de la vieille ville tout en reliant le port, la ville et les industries. II distingue les zones industrielles et résidentielles entre elles - les quartiers extérieurs sont les uns destinés aux ouvriers des industries portuaires, les autres aux employés et salariés à hauts revenus -, fonctionnalisant ainsi les données héritées de l'histoire urbaine. Les préoccupations de cette lecture fonctionnelle, reprises en 1940 par Eugène Beaudoin, et continuées par Georges Meyer-Heine ${ }^{7}$ à partir de 1949, sont finalement de trois ordres: rattraper les retards accumulés en terme d'équipements, inscrire la croissance urbaine dans un zonage fonctionnel et façonner une agglomération où les infrastructures sont conformes à la vocation portuaire de la ville. Les données urbaines d'une ville étendue et complexe, où le labyrinthe des rues du centre paraît contrarier la prospérité portuaire, en ressortent simplifiées; Toutefois, en dépit des urgences issues de la guerre et du retard en termes d'infrastructures, le manque de moyens, le climat d'instabilité politique entre 1947 et 1953, l'absence d'articulation entre les acteurs de la Reconstruction (urbanistes, architectes, etc.) ne permettent guère d'applications conséquentes de ces projets.

\section{Les premiers pas de la sociologie urbaine à Marseille}

C'est dans ce contexte que les premiers travaux de sociologie urbaine au sortir de la guerre, renversant la perspective fonctionnaliste, mettent la vieille ville, ouvrière et portuaire, au centre de leurs enquêtes. Réalisées par des prêtres ouvriers issus du mouvement "Économie et Humanisme », elles portent la marque de la tradition catholique d'enquête, de son souci de modernisation économique et de rationalisation sociale, de sa pratique monographique du terrain et de sa volonté de susciter l'instauration, par l'État et le patronat, de politiques sociales favorables à la classe ouvrière. Ses objets (hygiène, conditions de travail, description des « taudis ») rappellent 
les problématiques initiées avant guerre à la section d'hygiène du Musée Social ${ }^{8}$. Fédérant des membres du patronat catholique "moderniste", des militants de la J.O.C. et du Mouvement Populaire des Familles, ces sociologues participent à la grande enquête sur l'habitat du Ministère de la Reconstruction lancée en 1945-1946 à Nantes, Lyon, SaintÉtienne et Marseille9 . Pour traiter ces enquêtes conformément à leur idéal d'objectivité empirique (recours au questionnaire, construction de diagrammes «en roue », etc.) ${ }^{10}$, Louis Joseph Lebret, fondateur d'Économie et Humanisme, crée à Marseille en juillet 1945, avec Jean Queneau (fondateur du Centre des Jeunes Dirigeants à Marseille) et Jacques Loew, l'IMSAC (Institut marseillais de statistique, d'analyse et d'application comptable) ${ }^{11}$.

6 Premier de ces sociologues urbains marseillais, Jacques Loew, avocat devenu dominicain, s'est installé en 1941 en mission ouvrière dans le quartier de La Cabucelle et publie en 1944 une thèse de sociologie urbaine ${ }^{12}$. L'analyse monographique du mode de vie et des conditions de travail des dockers, qui emprunte parfois à Le Play, est au service d'une analyse urbaine. Une cartographie précise de l'habitat ouvrier est interprétée à la lumière de la gestion de la main-d'œuvre portuaire (emploi à la demi-journée au gré du trafic) qui commande la proximité entre l'habitat et le port au risque de l'entassement et de l'insalubrité ${ }^{13}$. Contrairement aux urbanistes, Loew refuse de considérer les vieux quartiers comme des lieux d'anomie et de dégénérescence faisant obstacle à la prospérité portuaire, mais au contraire se propose d'en décrire les formes spécifiques de sociabilité et de solidarité. L'empathie missionnaire devient regard ethnologique.

7 Ce travail reste néanmoins une exception. Les enquêtes sociologiques qui lui succèdent n'articulent plus guère l'observation ethnographique d'un quartier et d'un groupe social à une analyse globale de la question urbaine. Plus neutres, inspirées d'une anthropologie culturaliste des besoins des populations, elles s'ajustent, à partir de 1953, à la politique urbaine de G. Defferre (politique de construction en série de HLM dites simplifiées, laissée au soin de ses alliés de la droite modérée). En 1957 une première évaluation sociologique, Les nouveaux ensembles d'habitations à Marseille, dirigée par un ingénieur, Lucien Cabaniols, rassemble des institutions idéologiquement assez proches : l'IRES (Institut de recherches économiques et sociales), l'équipe "Urbanisme » du mouvement "La Vie nouvelle ", l'ALOGEC (Association pour le logement économique), et le Carrefour (association familiale marseillaise) se sont groupés sous l'appellation «Ville Humaine » pour «tenter de décrire succinctement les conditions d'habitat et la consistance des équipements collectifs existant dans tous ces groupes d'habitations de plus de cinquante logements construits à Marseille depuis la fin de la guerre. [...] Un tel travail pourrait apporter une vue utile à l'ensemble de tous les professionnels de la construction et de l'urbanisme ${ }^{14}$ ".

$\mathrm{Du}$ militantisme catholique de la Reconstruction, l'enquête conserve l'empirisme quantificateur (tableaux et diagrammes représentant la qualité de l'équipement pour chaque ensemble construit). Le recours aux sciences sociales semble un moyen de dépolitiser les enjeux urbains, "en allant sur le terrain sans autre souci que la photographie de la réalité [...] dégager l'opinion des familles sur leurs conditions de vie [... ] et préciser dans quelle mesure les équipements collectifs existants étaient adaptés aux besoins actuels et futurs de leurs usagers ${ }^{15}$." L'enquête repose sur un questionnaire constitué de questions de positionnement sur une échelle de satisfaction portant sur les problèmes d'insonorisation, d'isolement, de qualité des équipements sanitaires, ainsi que sur les besoins en équipements et services collectifs. L'unité d'analyse est "l'unité de voisinage ", catégorie spatiale définie à la fois comme une concentration de logements et de besoins en équipements (administratifs, sportifs et commerciaux), et comme une 
sphère de sociabilité qui exige certaines conditions d'environnement pour générer une vie sociale non pathologique. L'analyse de ces unités par catégories socioprofessionnelles montre qu'en dépit de la prédominance des ensembles dits «logécos» (constructions primées par un financement du Crédit Foncier), les CSP supérieures sont majoritairement logées en constructions « luxes », les CSP correspondant aux couches moyennes salariées en HLM aux normes élevées, alors que les CSP modestes sont les seules à occuper les HLM à normes réduites et les cités d'urgence. L'enquête indique que plus on s'éloigne du centre, plus nombreuses sont les constructions de bas standing. Cependant, l'unité retenue et le découpage géographique adopté (par zones concentriques et non par zones fonctionnelles ou par caractéristiques sociales) rendent invisible la différenciation entre l'urbanisation résidentielle au sud et ouvrière au nord, occultant le rôle joué par la proximité des zones industrielles.

\section{Changement d'échelle de l'intervention urbaine, changement d'objet pour les sciences sociales}

9 Au milieu des années soixante, d'importants projets d'aménagement de Marseille et de sa région constituent le contexte et parfois le prétexte d'un renouvellement des enquêtes et des questions posées par la recherche urbaine. Le projet d'aménagement de l'aire métropolitaine marseillaise (suscité par la DATAR, créée en 1963) combine le développement portuaire de Fos-sur-Mer et celui d'un « centre tertiaire directionnel » à Marseille. À cette fin, l'État met en place de nouveaux outils de planification urbaine (le SDAU, le SDAMM, etc.) $)^{16}$ et suscite de nouvelles institutions (l'OREAM, l'AGAM, etc.) ${ }^{17}$. Ce changement d'échelle géographique pose le problème de la place de Marseille dans une vaste région urbaine qui distingue espaces industriels (Fos-sur-Mer, étang de Berre) et tertiaires (Marseille) avec la perspective d'un développement urbain autour de l'étang de Berre ${ }^{18}$. La recherche urbaine locale, en même temps que s'accroît la part des financements publics (avec le développement d'une recherche contractuelle du CNRS) et que se modifie le profil des chercheurs (développement des départements universitaires de sociologie, d'ethnologie et d'urbanisme), recompose ses objets autour de ces évolutions, notamment en multipliant les enquêtes sur le centre-ville marseillais ${ }^{19}$.

$\mathrm{Au}$ moment où le projet de centre directionnel se met en place et avec lui une réflexion sur la fonction urbaine du centre marseillais, des enquêtes liées au SDAU (qui attribue au centre des fonctions : commandement, commerces, etc.), vont confirmer ou au contraire critiquer la pertinence d'une structuration urbaine qui combine promotion d'un centre tertiaire, développement de pôles urbains secondaires, et mise en place d'une politique de rénovation du centre par l'intermédiaire des premières ZAD (celles du Panier et de Belsunce notamment). Dans la logique des schémas directeurs, le travail conduit en 1968 par l'équipe de P.-H. Chombart de Lauwe ${ }^{20}$, en complément d'une vaste enquête conduite sur Paris ${ }^{21}$, analyse le rôle du centre et sa perception par différentes catégories sociales de citadins. Au moyen d'un questionnaire d'opinion et de pratiques, il décrit les comportements effectifs des habitants dans leurs rapports avec le centre et avec certains équipements de la banlieue. Bien que rédigé en forme de mise en garde contre l'uniformité rationnelle de la planification, les indexations de lieu sont rares et les conclusions sont toujours d'une extrême généralité. L'enquête reprend d'ailleurs, pour construire ses résultats, la partition de la ville en zones concentriques (centre et trois 
couronnes) occultant ainsi les phénomènes de ségrégation urbaine et les différences sociales d'usage de l'espace.

11 Repenser sociologiquement le centre-ville va justement consister pour la recherche urbaine à déconstruire cette vision fonctionnelle qui articule son réaménagement à sa fonction supposée d'attraction. Pour ce faire, c'est l'objet centre, construit implicitement sur le modèle de l'Agora, qui est reconstruit: au-delà de ses fonctions, le centre est un lieu de transit et d'acculturation. Ainsi Sylvia Ostrowetsky et Samuel Bordreuil ${ }^{22}$ l'interprètent comme un distributeur sur l'ensemble de l'agglomération. L'objet pertinent n'est plus le centre mais les centres spécialisés qui connectent des flux. La dimension interactive du lien entre le social et le spatial, référence à Raymond Ledrut, et la notion de multicentralité (qui confirme la nécessité de multiplier les centres périphériques, conformément aux orientations du SDAU) conduisent à un cadre d'analyse où Marseille est lue à travers des pratiques circulatoires, constituant ainsi une des premières critiques de l'idéalisme urbanistique.

Mais ce sont les analyses de Marcel Roncayolo sur la construction et la division sociales de l'espace marseillais à la fin des années soixante qui contribuent le plus à renouveler les méthodes de la recherche urbaine ${ }^{23}$. Analysant la répartition des activités, il montre que le centre, loin de concentrer tous les services tertiaires, conserve encore des activités quasi industrielles ainsi qu'une fonction résidentielle. Son originalité fonctionnelle (compte tenu de l'existence d'Aix-en-Provence) tient à la prédominance de la fonction commerciale, renforcée par l'emprise territoriale du port, et au fait que les fonctions administratives n'y sont pas prépondérantes. Contre la facilité d'un zonage fonctionnel, Roncayolo propose de « déceler l'action des groupes sociaux liés à cette économie, bref de passer des fonctions à la société ». L'analyse du ratio emplois/résidents et celle de la localisation des activités lui permettent de mettre en évidence deux pôles centraux : au nord l'influence portuaire et populaire et au sud celle des quartiers à connotation bourgeoise. Pour interpréter ce phénomène de double centralité, Roncayolo mène une double démarche, historique ( « replacer le centre et son évolution dans les courants qui ont orienté la progression urbaine») et écologique ("analyser les rapports entre localisation des fonctions, types d'habitat et structures sociales de la population résidente »). À partir des données de recensement, il distingue au sud ce qui relève des fonctions centrales (commerce rare, banque...) et consolide l'habitat bourgeois, et au nord les dégradations de quartiers abandonnés à une "société particulière, presque "autonome", à l'intérieur de la société marseillaise». Ce sont donc les logiques de l'habitat, de la composition sociale et ethnique et de la situation par rapport au port qui décrivent le mieux un centre qui a essentiellement une fonction d'acculturation ${ }^{24}$.

Marseille est ici un laboratoire urbain où s'éprouvent des méthodes de technicité inégale, selon différentes échelles d'observation. L'analyse factorielle de la géographie la plus quantitative permet ainsi de hiérarchiser les logiques qui produisent des ségrégations ${ }^{25}$. Les facteurs explicatifs des plus grands écarts sont les oppositions entre les strates sociales extrêmes (professions libérales et cadres supérieurs vs ouvriers), ensuite entre les commerçants et artisans vs les ouvriers et les contremaîtres, enfin entre la classe moyenne salariée et les autres catégories sociales. La combinaison entre les facteurs permet de construire une typologie (que l'on peut cartographier) qui précise la nature et l'évolution dans le temps de la division sociale de l'espace. Mais c'est la combinaison de démarches historiques, écologiques et quantitatives qui permet de déterminer comment cette division sociale s'atténue ou se renforce, selon quels clivages et quelles formes 
spatiales, et en quoi le changement d'échelle (le recours à des unités géographiques plus précises et plus concrètes) confirme le jeu des relations mises en évidence. L'analyse passe de la statistique à l'étude des processus de construction de l'espace. C'est l'évolution de la ville (poussée démographique depuis 1954, construction des grands ensembles de banlieue, déclin industriel, activités tertiaires en hausse, plus variées mais moins polarisées) qui a rompu la liaison activités-résidences essentielle au maintien des classes sociales élevées au centre. Du coup, le centre prolétaire, zone d'acculturation, a toutes les chances de devenir zone de ségrégation sans que soient remis en cause les rapports spatiaux. "Les nouvelles générations de cadres salariés constituent un groupe socioculturel très différent, souvent moins lié par ses origines géographiques à la tradition marseillaise [...]. La notion de "centre" n'est pas modifiée seulement par les nouvelles possibilités techniques ou les nouvelles exigences (circulation, espace, confort): elle est d'origine socioculturelle. La conception de l'espace urbain s'inscrit dans un certain style de vie. Malgré les forces unifiantes de la "société de consommation", cette conception n'évolue pas au même rythme, ni de la même manière, selon les groupes sociaux. L'attitude des cadres supérieurs à Marseille est significative de ces décalages ${ }^{26}$. "

À partir de démarches statistiques, écologiques et historiques, la sociologie et la géographie urbaines éclairent ici des évolutions de longue période, socialement déterminées et non réductibles à la seule adaptation fonctionnelle à des données techniques. À l'inverse, les sciences sociales peuvent être fondées à chercher ailleurs géographiquement, ou à un autre niveau de phénomènes sociaux, les logiques des transformations urbaines. Ainsi dans l'approche de la sociologie marxiste ${ }^{27}$, c'est moins la ville en tant que telle que la politique urbaine, interprétée comme une conséquence de la coopération entre les grandes entreprises nationales «monopolistes » installées autour de l'étang de Berre, la bourgeoisie marseillaise et l'État, qui est au centre des enquêtes. Celles-ci se donnent comme double objet les nouvelles formes et échelles du marché du travail et la politique urbaine marseillaise. L'analyse se veut localisée mais non monographique, Marseille étant le cadre d'étude d'un « système hégémonique local ». La politique urbaine conduite de 1953 à 1977 (période de l'alliance Defferre-bourgeoisie locale) s'expliquerait par les interdépendances entre l'implantation régionale du capitalisme "monopoliste", l'adoption d'une gestion technocratique de l'urbain (dépolitisation des enjeux urbains par technicisation du discours planificateur) et l'évolution du patronat local. La politique de G. Defferre est analysée comme un moment de dépolitisation de la politique urbaine, réduite à une politique d'infrastructures ${ }^{28}$ et à la promotion d'une clientèle tertiaire salariée. Le développement de "l'industrialisation polarisante » autour de Berre et Fos-sur-Mer, parce qu'elle tend à accentuer l'opposition au sein de la main-d'œuvre entre salariés des grandes firmes et salariés des entreprises sous-traitantes, et au niveau du capital entre firmes « centrales » et firmes "satellites », génère à la fois une segmentation du marché du travail et une dispersion résidentielle à l'échelle de l'aire métropolitaine. C'est dans cette perspective que sont conduites les premières enquêtes sur les mobilités au sein du territoire métropolitain ${ }^{29}$.

15 Les grandes opérations d'aménagement des années 1965-1975 suscitent donc des enquêtes urbaines d'une grande diversité méthodologique et idéologique. La chronologie qui résulte d'une confrontation des contextes de la politique et de la recherche urbaines, tant au niveau national que local (ce que suggère la chronologie en annexe) permet de décrire les oppositions et successions propres à une sociologie de la sociologie urbaine. Mais audelà des effets de conjonctures institutionnelle ou idéologique, elles témoignent, à 
l'exemple des travaux sur la centralité, de la manière dont Marseille a pu être non seulement le terrain singulier d'applications localisées de cadres théoriques élaborés par ailleurs ${ }^{30}$, mais aussi le champ d'application de nouvelles méthodes d'investigation (recours aux archives cadastrales, aux recensements, à la sociologie électorale) et de traitement (représentation des flux de circulation, analyse factorielle, jeu sur les découpages d'unités spatiales, usage de la sémiologie graphique dans la cartographie), concourant ainsi au renouvellement des sciences sociales urbaines.

\section{Crise du système urbain et intervention localisée de la recherche}

$\mathrm{Au}$ cours des années quatre-vingt, la crise de l'économie et du système urbain marseillais et la transformation du contexte institutionnel de la recherche liée au renouvellement du cadre législatif ${ }^{31}$ et à la décentralisation (diversification des financements locaux de la recherche) vont conduire la recherche urbaine à changer de cadre d'analyse et d'échelle d'observation. Elle passe d'analyses globales de l'offre urbaine à des enquêtes localisées et à des analyses micro-sociales des demandes et des modes de vie des habitants. Elle prend pour objet, directement ou indirectement, les politiques urbaines, moins pour en évaluer l'efficacité que pour mettre en évidence leurs présupposés inconscients ou leurs effets non voulus. De ce fait, les micro-contextes - que ce soit ceux du face à face logeurs/ habitants ou des interactions politico-administratives - deviennent les objets d'une pratique de recherche souvent expérimentale où la sociologie construit ses interprétations dans la déconstruction localisée des regards sur le quartier ou sur la ville.

Ainsi, Michel Anselme et Michel Péraldi développent avec l'équipe du CERFISE réunissant sociologues, techniciens et architectes, une "maitrise d'œuvre sociale ${ }^{32}$ " dans le cadre des opérations de réhabilitation de la cité du Petit Séminaire à Marseille ${ }^{33}$. La démarche d'enquête privilégie la saisie directe, au contact des habitants, du concret de la vie de la cité. Elle ne consiste pas à construire un protocole d'enquête à partir des problématiques de la réhabilitation mais à travailler sur. la communication entre logeurs et habitants et à favoriser l'émergence d'un espace public (une permanence hebdomadaire dans un lieu neutre où toutes les demandes concernant la réhabilitation doivent être publiquement verbalisées) ${ }^{34}$. La singularité de cette sociologie expérimentale tient à la position d'intermédiaire quasi inconscient du chercheur qui ne cherche pas à maitriser l'enquête. Ce n'est qu'une fois le travail accompli qu'un récit d'interprétation peut s'écrire. L'attention aux détails les plus anodins du quotidien, la relative discrétion et le flou de la position institutionnelle sont revendiqués comme les voies par lesquelles la maîtrise d'œuvre sociale parvint à déplacer les positions de chacun dans le face-à-face logeurlocataires et à déconstruire certains schèmes de perception de ces cités paupérisées. La maîtrise d'œuvre sociale, en posant à l'échelle micro-locale la question de la territorialisation des services urbains, a montré la nécessité de faire participer les habitants à la requalification de leur quartier, résultat qui a trouvé quelques prolongements dans le cadre de la politique DSQ.

Ces nouvelles procédures d'intervention urbaine deviennent aussi des objets d'investigation propres. Ainsi, l'histoire entre 1981 et 1987 de l'opération DSQ menée sur le quartier Belsunce ${ }^{35}$, composante de l'hypercentre marseillais caractérisée par une population maghrébine et pauvre et un habitat très dégradé, permet-elle à Philippe 
Chaudoir et Marie-Hélène Poggi de retracer les évolutions de l'équilibre institutionnel et des choix politiques qui font passer d'une logique de développement social du quartier comme espace délimité (objet d'OPAH successives) à une logique urbanistique de déstigmatisation du centre. À l'objectif initial d'aménager avec la population résidente son espace en le réhabilitant, succède, avec la création en 1985 de la «mission centreville ", une politique dont le secteur d'intervention devient moins le quartier que l'hypercentre, et dont l'objectif est désormais de réadapter le centre à ses fonctions «traditionnelles » en accueillant de nouveaux habitants ${ }^{36}$.

Au-delà de l'analyse d'une politique publique, l'étude de la construction sociale des populations des quartiers défavorisés par des enquêtes sur la procédure d'attribution des logements ${ }^{37}$ montre comment, avec l'arrêt de la construction et l'immobilisation des couches sociales les plus défavorisées, on est passé de critères normatifs et abstraits d'accession au logement social à des critères basés sur la composition sociale et la sociabilité locale. Avec la décentralisation des procédures d'attribution, cette évolution entraîne une contextualisation permanente, chaque grand ensemble immobilier étant l'objet d'un double diagnostic du candidat et du quartier. C'est donc le micro-contexte politico-administratif et les réseaux notabiliaires locaux qui deviennent les objets pertinents d'un travail expérimenté à Marseille (à Saint-Barthélemy) où le sociologue et l'habitant sont encore partenaires; le premier comme expert observe les processus d'interaction entre les parties concernées, le second sollicité par les associations de locataires donne son avis sur les cohabitations « acceptables».

Les contextes localisés du travail de construction, de stigmatisation ou d'occultation des quartiers de pauvreté deviennent donc des objets spécifiques de la recherche urbaine. L'analyse des phénomènes sociaux désignés sous le terme de "crise» et celle des dispositifs institutionnels prévus pour y répondre permettent de déconstruire l'image négative des grands ensembles. Rapportée aux enjeux inavoués des opérations de réhabilitation - faire revenir les classes moyennes, synonymes de bons locataires, et tenter d'en faire partir les populations captives, censées être à l'origine de la fuite des premiers -, la déconstruction du discours commun qui oppose population captive/ population qui fuit, immobile/mobile, illégitime/légitime ${ }^{38}$ indique comment le sens commun urbanistique a pu trouver là un principe d'explication qui économise d'analyser la réalité des trajectoires professionnelles, familiales et résidentielles des classes moyennes dont la cité n'a représenté qu'une étape et dont on entretient l'illusion du retour.

21 Aux analyses en termes de crise économique et sociale, ces diverses enquêtes, plus localisées, et souvent expérimentales, ont opposé dans leurs interprétations celles d'une " crise du regard » urbanistique. Le doute de la pensée technocratique sur sa capacité à maîtriser les conditions de l'ajustement entre un contexte urbain et des formes de développement économique et social a ainsi servi de prétexte heureux à la mise en place de nouveaux cadres de recherche, expérimentaux, localisés et attentifs au poids du lieu.

\section{Flux, migration, territoires identitaires}

Les recherches conduites sur Marseille et sa région, au fur et à mesure qu'elles souhaitaient rendre compte de phénomènes plus complexes, ont ainsi de moins en moins consisté à prendre la mesure des effets des politiques urbaines et de plus en plus à s'interroger sur les effets des logiques propres aux pratiques urbaines des citadins. On a 
ainsi assisté progressivement à un renversement des logiques de recherche, la production de la ville - la structuration de l'aire métropolitaine marseillaise -, étant interprétée à partir des dynamiques territoriales des populations. Ce changement d'objet commande un changement de méthodes et en particulier un recours aux descriptions des pratiques de l'espace et des réseaux sociaux.

Ainsi, l'analyse conduite par Alain Tarrius ${ }^{39}$ des dynamiques territoriales de différents groupes de migrants privilégie-t-elle l'examen de trajectoires socio-spatiales complexes : elle part de l'exploration approfondie des pratiques de mobilité, propres à une souspopulation singularisée (par hypothèse) par une identité professionnelle ou culturelle bien précise, qui sont au fondement de pratiques urbaines spécifiques. Dans le cas des familles lorraines immigrées en 1973, autour du nouveau pôle sidérurgique de Fos-surMer, il montre qu'en dépit des programmes de logements sociaux prévus pour accueillir les sidérurgistes dans les communes environnantes, les Lorrains, dès 1975, partent construire leurs résidences dans la plaine désertique de la Crau, dans les Alpilles ou sur la Côte Bleue. Ce groupe connaît ainsi la plus grande extension territoriale parmi les populations de l'ouest métropolitain marseillais, autour de pôles qui structurent ce territoire: la zone sidérurgique de Fos-sur-Mer pour le travail, Martigues pour la réactivation du lien identitaire et Saint-Martin-de-Crau pour l'habitat, lieu du statu quo entre Lorrains et sociétés locales.

Étudiant selon la même méthode les entrepreneurs commerciaux maghrébins, A. Tarrius part de 37 familles du quartier Belsunce, elles-mêmes liées à un deuxième ensemble de 838 personnes localisées dans la ZUP nord et ses environs, et à un troisième de 5026 personnes, décrivant ainsi le réseau d'une vaste métropole maghrébine fortement organisée, parcourue d'incessants mouvements et dont Belsunce est en quelque sorte le comptoir colonial. Trois espaces structurent ce système : la ZUP nord, relais des échanges entre Belsunce et la région; les villes des rives de l'Étang de Berre et leurs vastes ensembles d'habitat social, le triangle Cavaillon-Saint-Rémy-Carpentras avec sa forte concentration de main-d'œuvre agricole qui permet aux commerces maghrébins de distribution alimentaire dans Marseille de s'approvisionner, et le triangle ManosqueSisteron-Digne marqué par un important élevage du mouton; enfin, l'Algérie, la Tunisie et le Maroc. La fonction de ces entrepreneurs échappe aux prévisions des aménageurs locaux et c'est l'investigation sociologique qui révèle les décalages entre les logiques de peuplement et celle des politiques locales.

Aux politiques volontaristes d'aménagement, la recherche urbaine a donc opposé des objets, parfois de petite taille, parfois débordant les découpages administratifs et territoriaux convenus ou confrontant les échelles d'observation. Elles ont ainsi permis une meilleure connaissance de la population qui vit dans les espaces périurbains ${ }^{40}$. Plus spécifiquement, les approches longitudinales (suivis actualisés de cohortes d'individus) ont un intérêt particulier à Marseille dans la mesure où elles analysent la ville à partir des dynamiques territoriales issues des populations migrantes, qu'il s'agisse des études sur la structuration de l'aire métropolitaine par les réseaux sociaux, ou par les diverses pratiques de mobilités (mobilités quotidiennes, migrations, modes de transports). À titre d'exemple, les accédants à la propriété en zones périurbaines, après avoir été l'objet d'analyses de la mobilisation de la famille au service d'un projet social ${ }^{41}$, ont été étudiés, dans le cas de Lançon-de-Provence, à partir des mouvements migratoires et résidentiels ; leur reconstitution par le sociologue permet de construire une histoire de l'espace local 
qui croise les dynamiques migratoires régionales, les processus d'urbanisation locale et l'évolution de la morphologie sociale ${ }^{42}$.

Cette socio-anthropologie de la mobilité est ainsi un descripteur des structurations de l'espace à partir de nouvelles pratiques urbaines, qui accentuent les distances entre pôles d'emploi et lieux de résidence, entre lieux de résidence et lieux de consommation, et qui sont à l'origine des déséquilibres régionaux contemporains. En revanche, appliquée à l'analyse du capitalisme maghrébin, l'approche insiste plus sur les caractéristiques de l'exclusion et de l'imperméabilité des frontières ethniques que sur la production de l'urbanité. Constituant des réseaux invisibles, ces « poor capitalists » marginalisent ceux qui jouent le jeu du marché marseillais, comme l'indiquerait la stricte distinction des clientèles entre le Centre Bourse et les commerces maghrébins de part et d'autre du cours Belsunce. À l'inverse des analyses de Marcel Roncayolo qui décrivaient la coexistence des groupes sociaux au-delà de la ségrégation (approche de la différence par la proximité en quelque sorte), les modes de la coexistence sont analysés comme s'ils étaient les mêmes que ceux de l'exclusion.

Au terme de ce parcours on est tenté d'opposer, après les premières enquêtes des années de la Reconstruction, d'un côté des travaux qui analysent la croissance urbaine à Marseille à partir de problématiques globales élaborées ailleurs et appliquées à Marseille (études sur les grands ensembles ou analyses critiques de la politique urbaine), et de l'autre des analyses plus anthropologiques, plus localisées et plus soucieuses de comprendre, voire de susciter des relations sociales "horizontales». Ces dernières années ont vu la multiplication de démarches de recherches très locales aux ambitions explicatives limitées. À l'opposition analyses globales-croissance $v s$ analyses locales-crise, s'ajoute, sans consécution claire, l'évolution du contexte institutionnel (en particulier la décentralisation) qui affecte la politique urbaine et les formes prises par le financement de la recherche.

Pourtant, au-delà de l'absence de cumulativité de ces contributions (pont aux ânes des critiques épistémologues des sciences sociales), la diversité de ces travaux est une invitation à voir Marseille, en tant que terrain urbain, comme le lieu où ont pu se croiser, d'une recherche l'autre, les objets et les échelles d'observation, à l'exemple de l'articulation entre l'analyse de la ségrégation urbaine et celle des logiques de mobilité (résidentielle et sociale) et de circulation dans l'aire métropolitaine marseillaise. Dans cette perspective, les urbanités marseillaises apparaissent comme propice à la confrontation entre les analyses des effets des choix techniques d'aménagement (on sait autour de l'étang de Berre combien un tracé autoroutier produit du territoire), les études sociologiques de morphologie sociale et les observations ethnologiques fines des pratiques de l'espace. Bref, un terrain urbain de l'enquête. 


\section{Chronologie}

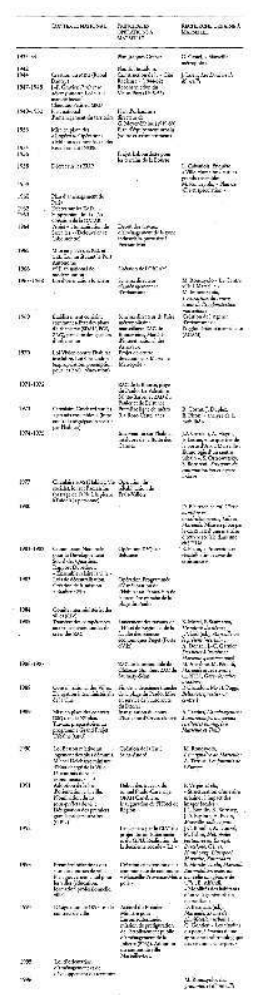

\section{NOTES}

1. Ce texte est issu d'une recherche effectuée avec S. Mazzella, Cinquante ans de recherche urbaine à Marseille. Bilan bibliographique (1945-1995), Paris, Secrétariat permanent du Plan urbain, 1995. Je remercie J.-P. Daviet, B. Morel et C. Paradeise pour leurs conseils.

2. À propos de la remarque de M. Halbwachs, «S'il existe à l'Université de Chicago une école de sociologie originale, cela n'est pas sans rapport avec le fait que ces observateurs n'ont pas à chercher bien loin leur objet d'étude » («Chicago, expérience ethnique » [1932], in Y. Grafmeyer et I. Joseph, eds, L'École de Chicago. Naissance de l'écologie urbaine [1979], Paris, Aubier, 1984, p. 291), G. Noiriel écrit: "Mais il y a en France, à l'époque, autant d'immigrés qu'aujourd'hui, et si Marseille n'est pas tout à fait Chicago dans les années trente, avec ses colonies juive, italienne, arménienne, nord africaine, espagnole... qui constituent une partie considérable de sa population (surtout si on tient compte de l'origine), la ville a tout du laboratoire ethnique; ce n'est donc pas l'objet qui fait défaut, mais le sociologue. » Le Creuset français, Paris, Seuil, 1988, p. 30.

3. J. Greber, Plan d'aménagement et d'extension de la ville de Marseille. Mémoire descriptif, Paris, Institut d'urbanisme-Vincent, Fréal \& cie, 1933. Voir également, R. Belhadj, Marseille et le projet Greber, 1933, thèse de doctorat, Paris, EHESS, 1990, multig.

4. En particulier G. Castel dans « Marseille-Métropole », Les Cahiers du Sud, 1934, et G. Rambert, Marseille: la formation d'une grande cité moderne. Étude de géographie humaine, Marseille, Maupetit, 
1934, ainsi que "L'extension de la ville de Marseille et le Plan Greber », Bulletin de la Société de Géographie de Marseille, LVI, 1935, p. 8-25.

5. La question ouvrière fut un des premiers objets des sciences sociales à Marseille, à travers les études leplaysiennes d'E. Rostand, Questions d'économie sociale dans une grande ville populaire, Paris, 1889. Voir M. Roncayolo, "Marseille "grande ville populaire", le regard de l'économie sociale ", Marseille, 164, 1992, p. 63-69. Sur la question des migrations, E. Temime, ed., Migrance. Histoire des migrations à Marseille, Aix-en-Provence, Edisud,

4 tomes, 1991.

6. M. Roncayolo, Les grandes villes françaises: Marseille, Paris, La Documentation française (« Notes et études documentaires ", 3013), 1963, p. 43. La ville surmonta cet excès d'extension grâce au tramway, réalisation du socialisme municipal de S. Flaissières (1891). C'est peut-être ce trait de morphologie, plus que la présence de populations immigrées (cf. note 2), qui suscita chez Roncayolo, lecteur d'Halbwachs, la comparaison entre Marseille et Chicago : "De même que les buildings ne seraient pas possibles sans les ascenseurs, on ne conçoit pas qu'une ville ait pu s'étendre à ce point sans se disperser tout à fait et en conservant quelque unité, sans les trains et les tramways électriques. » (M. Halbwachs, « Chicago, expérience ethnique », op. cit., p. 290.)

7. G. Meyer-Heine, « Le Plan Directeur », Marseille, 13, 1951.

8. Voir, par exemple, le travail du professeur J. Trémolières, «Enquête sur l'alimentation et les dépenses des familles de dockers de Marseille ", Bulletin de l'Institut national d'hygiène, VII, janviermars 1952, p. 64-95. Nutritionniste, il est un collaborateur régulier de l'équipe de Chombart de Lauwe à la fin des années cinquante. Sur l'idéologie de cette sociologie urbaine, cf. P.-P. Zalio, «La renaissance des études urbaines en France dans la période de Reconstruction », mémoire de DEA, Paris, ENS, 1989, multig., p. 50-56, ainsi que I. Astier et J.-R. Laé, « La notion de communauté dans les enquêtes sociales sur l'habitat en France, 1945-1955 ", Genèses, 5, 1991, p. 81-106.

9. Pour une analyse similaire, cf. M. Quoist, La ville et l'homme. Rouen, étude sociologique d'un secteur prolétarien suivi de Conclusions pour l'action, préface de G. Le Bras, Paris, Éd. du CNRS-Éditions ouvrières-Économie et Humanisme, 1952.

10. Sur ce recours à l'objectivité empirique, et la construction de ces diagrammes cf. L.-J. Lebret, Guide pratique de l'enquête sociale, vol. 3, L'enquête urbaine. L'analyse du quartier et de la ville, Paris, Presses universitaires de France, 1955.

11. Comme le note J. Ion, «l'activité scientifique n'est pour eux qu'un moyen au service de fins militantes, le passage pour conquérir une position d'énonciation, même si l'enthousiasme de l'idéal objectiviste les conduit souvent à sans cesse complexifier les instruments d'observation du social qu'ils utilisent ou mettent au point ", J. Ion, " "Ingénieurs sociaux" et sciences sociales appliquées, le groupe Économie et Humanisme de Saint-Étienne de 1944 à 1953 », Économie et Humanisme, 307, 1990.

12. J. Loew, Les Dockers de Marseille. Analyse type d'un complexe social, préface de G.Thibon, L’Arbresle, Éd du CNRS-Économie et Humanisme, 1944.

13. «Les entassements de certaines familles dans des enclos misérables à quelques centaines de mètres du port sont la conséquence fidèle de l'organisation du travail. [...] Supprimer le taudis consiste à changer d'abord le mode d'embauche." J. Loew, En mission prolétarienne, Paris, Économie et Humanisme, 1946, p. 26.

14. L. Cabaniols, L'habitat et l'équipement collectif, Marseille, IRES-Ville Humaine, 1960, p. 12.

15. L. Cabaniols, ibid., p. 13.

16. SDAU : Schéma directeur d'aménagement et d'urbanisme de l'agglomération marseillaise (1969). SDAMM : Schéma d'aménagement de l'aire métropolitaine marseillaise (1967).

17. OREAM : Organisation pour les études d'aménagement de l'aire métropolitaine marseillaise (1966). AGAM : Agence d'urbanisme de l'agglomération marseillaise (1969).

18. Une des conséquences de ce zonage, alors que disparaissent les industries marseillaises, est la déconnexion économique progressive de Marseille par rapport à sa région, et finalement sa 
paupérisation. Sur ce point, B. Morel et P. Sanmarco, Marseille, l'endroit du décor, Aix-en-Provence, Edisud, 1983.

19. Les premiers travaux sur le centre furent l'œuvre des urbanistes qui l'envisageaient plutôt comme un « kyste ", les vieux quartiers étant vus comme une barrière séparant le port de la ville «moderne ». Plus que d'études, il s'agissait soit de projets de remodelage associant destructions et réaffirmation d'une vocation de convergence, soit d'enquêtes d'hygiène et de salubrité. Le centre devient désormais un objet tant sociologique qu'ethnologique, voir J. A. Carreno, A. Hayot et F. Lesme, "Ethnologie d'un centre urbain : le quartier de la Porte d'Aix à Marseille ", Paris, Musée de l'Homme, 1974.

20. M. Imbert, B. Lamy, N. Petrenko et J. Retel, L'attraction du centre. Étude sociologique de l'agglomération marseillaise, Paris, Centre de sociologie urbaine/Commissariat général du plan, 1968.

21. P.-H. Chombart de Lauwe, ed., L'attraction de Paris sur sa banlieue, Paris, Éditions ouvrières, 1965.

22. S. Ostrowetsky et S. Bordreuil, Structure de communication et espace urbain : la centralité, Paris, Direction de l'aménagement foncier et de l'urbanisme, 1975.

23. M. Roncayolo, «Le centre-ville à Marseille : notion, contenu, évolution ", in Urban Core and Inner City, Leyden, University of Amsterdam, E. J. Brill, 1967, p. 162-182. Pour une vue complète de cette contribution majeure, il faut se reporter à sa thèse (1981), Les grammaires d'une ville. Essai sur la genèse des structures urbaines à Marseille, Paris, Éd. de l'EHESS, 1996.

24. M. Roncayolo peut alors interpréter des formes urbaines concrètes comme la Canebière, décrite comme un "thalweg ": «Dans un milieu social où la ségrégation n'est jamais poussée à son extrême, la Canebière n'appartient pleinement à aucune des aires fonctionnelles ou sociales que nous avons définies : elle en est la limite, mais aussi le front de chevauchement, l'accès. Cet axe redonne ainsi une unité organique à la fois aux divers compartiments entre lesquels se fractionne le centre, et à la ville tout entière. " M. Roncayolo, « Le centre ville à Marseille », op. cit., p. 177.

25. M. Roncayolo, "La division sociale de l'espace urbain. Méthodes et procédés d'analyse ", Bulletin de l'Association des Géographes français, 395-396, 1972, p.5-20, 31-35. Également, Les grammaires d'une ville..., op. cit., p. 275-299.

26. M. Roncayolo, « Le centre-ville à Marseille », op. cit., p. 179 et 182.

27. D. Bleitrach, J. Lojkine, E. Oary, R. Delacroix et C. Mahieu, Classe ouvrière et social-démocratie. Lille et Marseille, Paris, Éditions sociales, 1981 ; A. Chenu, Industrialisation, urbanisation et pratiques de classe. Le cas des ouvriers de la région marseillaise, thèse de sociologie, Université de Toulouse, 1981, multig.

28. Le projet de métro (1964) a ainsi vocation à « relier la zone centrale où doit se localiser le "tertiaire supérieur" aux périphéries où résident les employés - la Rose, les Caillols - et les cadres - Mazargues ", D. Bleitrach, op. cit., p. 148.

29. L'analyse des déplacements ne porte pas seulement sur les migrations pendulaires que l'amélioration du réseau autoroutier contribue sans cesse à intensifier. Il s'agit d'identifier les conséquences de la désindustrialisation de Marseille et du développement des grandes entreprises et de leurs sous-traitants autour de l'étang de Berre, c'est-à-dire de la segmentation du marché du travail. La politique d'infrastructures routières y est interprétée comme un transfert de plus-value. Voir R. Cornu, J. Duplex et B. Picon, "Analyse contextuelle de la mobilité », CNRS-LEST-CORDES (1973), et D. Bleitrach et A. Chenu, Espace urbain, transports et mode de vie, contrat DGRST, Université d'Aix-Marseille II, 1980, multig.

30. Le «système Defferre » a ainsi servi à la sociologie urbaine marxiste de cas historique d'un type de «système hégémonique local » qui dans ses traits singuliers - Marseille n'étant pas une «Company town» comme la monopolville dunkerquoise - contredisait assez largement le modèle gramscien de l'hégémonie bourgeoise dont il se devait d'être une déclinaison. 
31. Loi sur l'accession à la propriété marquant le passage de l'aide à la pierre à l'aide à la personne (1977), circulaire habitation et vie sociale (HVS, 1977), prime à l'amélioration du logement à usage locatif à occupation sociale (PALULOS), opérations programmées d'amélioration de l'habitat (OPAH, 1982). Deux OPAH sont ainsi lancées dans le quartier Belsunce. Les quartiers du Panier et de Belsunce bénéficient aussi de la loi Vivien (1970) sur la résorption de l'habitat insalubre (RHI) qui oblige, après rénovation, à reloger les habitants dans un secteur proche. Cinq RHI sont conduites dans les années quatre-vingt dans le quartier du Panier, représentant 78 immeubles démolis ou à démolir et la création de 210 logements sociaux. Au début des années quatre-vingt, est mise en place la procédure de développement social des quartiers (DSQ). Cette procédure, contractualisée pour la région PACA en 1984 dans le cadre du IX e plan, est appliquée dans les quartiers du Panier et de Belsunce.

32. M. Anselme et M. Péraldi, "Le Petit Séminaire à Marseille», Annales de la Recherche urbaine, 26, 1985 ; M. Anselme, «Situations d'expertise et intervention sociologique : le cas des maîtrises d'œuvre sociale dans quelques opérations de réhabilitation des cités HLM de Marseille », table ronde CRESAL, Situations d'expertise et socialisation des savoirs, Saint-Étienne, 1985.

33. Construite en 1959 par l'OPHLM de Marseille, la cité du Petit Séminaire est alors habitée par une population pauvre composée de Français d'origine méditerranéenne, d'immigrés algériens et tunisiens et de gitans d'origine andalouse ; de nombreux logements demeurent vacants alors que la demande des familles ne cesse d'augmenter dans cette cité. Une opération de réhabilitation est menée en concertation avec les habitants de 1980 à 1985.

34. M. Anselme et M. Péraldi, « Le Petit Séminaire à Marseille », op. cit., p. 64.

35. P. Chaudoir et M.-H. Poggi « Belsunce, quartier ou centre? », in Politiques urbaines ou politiques sociales, Aix-en-Provence, CERPE, 1987. Sur Belsunce voir E. Temime, Marseille transit. Les passagers de Belsunce, Paris, Autrement, 1995, ainsi que S. Mazzella, L'enracinement urbain. Intégration sociale et dynamiques urbaines, thèse de doctorat, EHESS, 1996, multig.

36. Dans un contexte de progression du Front national, nombre de gestionnaires et d'élus pensent qu'en cherchant à revaloriser le quartier Belsunce la politique DSQ n'a fait qu'entretenir l'éclatement de la centralité.

37. C. Dourlens, P. Vidal-Naquet, L. Henck-Pierrot, P. Chaudoir et M.-H. Poggi, Gestion et sujétion. À propos des stratégies de requalification sociale des quartiers de Marseille, Marseille, CERPE, 1987.

38. M. Anselme, «La formation des nouveaux territoires urbains et leur crise : les quartiers nord de Marseille », in Gérer les crises urbaines, Marseille, 1987.

39. A. Tarrius, Identités sociales et usages des temps et des espaces dans les périphéries urbaines. Mobilités et territoires lorrains dans la région de Fos-sur-Mer, Paris, INRETS/Plan Urbain, 1987, ainsi que Les fourmis d'Europe. Migrants riches, migrants pauvres et nouvelle ville internationale, Paris, L'Harmattan, 1992.

40. Ainsi, par exemple, les travaux dirigés par P. Vergès sur le rôle des identités locales dans la structuration de la zone de l'étang de Berre: $\mathrm{P}$. Vergès, Modes de vie et déplacements des habitants des rives de l'étang de Berre, EPAREB, 1989, ou, plus récemment, les travaux du laboratoire LAMES (CNRS-Université de Provence), comme ceux d'A. Donzel sur la coopération intercommunale.

41. N. Spinouza, La propriété différée. Les accédants à la propriété en difficulté dans les Bouches-duRhône, Marseille, CERFISE, 1991.

42. E. Affandi, "Mobilités des habitants d'un village périurbain marseillais ", Annales de la Recherche urbaine, 59-60, 1993, p. 100-108. 


\section{RÉSUMÉS}

Le parcours de cinquante ans de recherche urbaine marseillaise permet de montrer que la diversité successive des problématiques et des objets de la sociologie urbaine ne s'y réduit pas à de simples ajustements, à des conjonctures institutionnelles et idéologiques nationales mais constitue Marseille en laboratoire urbain. La pluralité des orientations de recherche témoigne ainsi d'une capacité, face à des problématiques souvent préconstituées par la demande sociale, à innover en terme de protocole d'observation (variation des échelles du quartier à l'aire métropolitaine, des situations d'interaction aux mobilités), de méthodes (du questionnaire de satisfaction au terrain ethnologique en passant par la géographie quantitative) et de catégorie d'analyse.

The course of fifty years of urban research on Marseilles reveals that the successive diversity of problematics and objects of urban sociology is not limited to simple adjustments to institutional conjunctures and national ideologies but have transformed Marseilles into an urban laboratory. Indeed, the mulitiplicity of orientations of research are thus proof of a capacity to innovate when confronted with problematics often preconstituted by social demand : in terms of the protocol of observation (variation of scales from neighbourhood to the metropolitan space, from situations of interaction to mobilities), of methods (from the questionnaire of satisfaction on the ethnological terrain by way of quantitative geography) and of analytical categories.

\section{AUTEUR}

\section{PIERRE-PAUL ZALIO}

Pierre-Paul Zalio (Ecole normale supérieure de Cachan) achève actuellement une thèse de sociologie sur le patronat marseillais au XX ${ }^{\mathrm{e}}$ siècle. Il a publié récemment : « Max Weber : économie et société, une typologie du pouvoir comme instrument de comparaison historique », in Le pouvoir, Paris, Ellipses, 1994, pp. 7-20 ; «Echec industriel et réussite sociale, le cas du capitalisme familial marseillais », in Entreprises et Histoire (Paris), n 9,1995, pp. 93-105 et, en collaboration avec S. Mazzella, Cinquante ans de recherche urbaine à Marseille, bilan bibliographique (1945-1995), Paris, Secrétariat permanent du plan urbain, 1995 